\title{
REVISIÓN DE DOUVILLININAE (BRACHIOPODA) DE LA ZONA CENTROIBÉRICA MERIDIONAL (DEVÓNICO SUPERIOR, ESPAÑA)
}

\author{
Miguel V. PARDO ALONSO \\ Departamento de Geología. Universitat de València. C/ Dr. Moliner 50. \\ E-46100-Burjassot (Valencia) ESPAÑA. e-mail: Miguel.V.Pardo@uv.es
}

Pardo Alonso, M.V. 2002. Revisión de Douvillininae (Brachiopoda) de la Zona Centroibérica meridional (Devónico Superior, España). [A revision of Douvillininae (Brachiopoda) from the southern Central-Iberian Zone (Upper Devonian, Spain)]. Revista Española de Paleontologia, 17(1), 157-164. ISSN 0213-6937.

\begin{abstract}
Frasnian Douvillininae in the southern Central-Iberian Zone are represented by the genus Douvillina with the three species Douvillina alvarezi, D. delta, and D. radiata. Given the external variability of many Douvillininae species, including the type-species $D$. dutertrei, an emendation to the Douvillina diagnosis stated in the revised edition of the Treatise on Invertebrate Paleontology (part H, Brachiopoda) is proposed herein. The emendation could allow the inclussion in Douvillina of specimens with very variable shell convexity from strongly concaveconvex to faintly plano-convex profile. The assignation of D. alvarezi to the emended genus is discussed and new, younger specimens than the type material are included in this species.
\end{abstract}

Keywords: Brachiopoda, Douvillininae, systematics, biostratigraphy, Devonian (Frasnian), CentralIberian Zone, Spain.

\section{RESUMEN}

La subfamilia Douvillininae en el Frasniense de la Zona Centroibérica meridional está representada por el género Douvillina, al que se atribuyen tres especies: Douvillina alvarezi, D. delta y D. radiata. Se propone enmendar la diagnosis del género Douvillina que aparece en la edición revisada del Treatise on Invertebrate Paleontolog. (Part H, Brachiopoda), en el sentido de admitir mayor variabilidad en la convexidad de las valvas. Se discute la atribución de $D$. alvarezi al género así enmendado y se incorporan a la especie ejemplares estratigráficamente más jóvenes que los del material típico.

Palabras clave: Brachiopoda, Douvillininae, sistemática, bioestratigrafía, Devónico (Frasniense), Zona Centroibérica, España.

\section{INTRODUCCIÓN}

En el Frasniense de la parte media de la Zona Centroibérica (ZCI) meridional (Fig. 1) es frecuente encontrar niveles fosilíferos con braquiópodos pertenecientes a la subfamilia Douvillininae Caster, 1939. En el sinclinal de Almadén, por ejemplo, Pardo y GarcíaAlcalde (1984) describieron, entre otras, tres especies nuevas asignadas a dos géneros de la subfamilia: Douvillinoides (?) alvarezi, Douvillina delta y Douvillina radiata; también citaron, pero sin describirla, una cuarta especie, Douvillina sp., común en los niveles de la biozona de Pradochonetes muelleri Pardo y García-Alcalde, 1984.

Nuevas investigaciones han ampliado el conocimiento de estos Douvillininae, advirtiéndose su presencia en gran parte del ámbito de la ZCI meridional. También se localizó más y mejor material en los niveles que contenían Douvillina sp., lo que permite precisar más su determinación.

Recientemente, Cocks y Rong (2000), en su revisión de los Strophomenida para el volumen 2 de la parte $\mathrm{H}$ (Brachiopoda) de la edición revisada del Treatise on Invertebrate Paleontology (T.I.P.), introdujeron novedades en la diagnosis de varios géneros, entre ellos Douvillina. Sin embargo, la morfología de las especies centroibéricas sugiere la necesidad de modificar de nuevo la diagnosis de dicho género en el sentido que se expresa a continuación. 


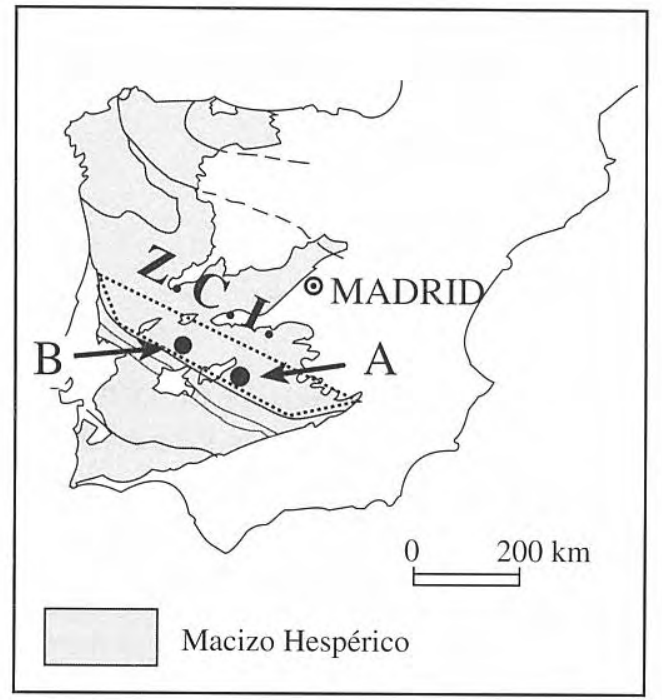

Figura 1. Localización geográfica y geológica de la Zona Centroibérica (ZCI) meridional (recuadro), en el Macizo Hespérico. A, parte central, con los sinclinales de Herrera del Duque, Almadén, Guadalmez y Pedroches. B, sinclinal de la Sierra de San Pedro.

Geographical and geological setting of the southern Central-Iberian Zone (CIZ) in the Hesperian Massif. A, central part: Herrera del Duque, Almadén, Guadalmez and Pedroches synclines. B, Sierra de San Pedro syncline.

\section{SISTEMÁTICA}

Todo el material citado y siglado se conserva en las colecciones de los museos siguientes (siglas entre paréntesis): Museo del Departamento de Geología de la Universitat de València (MGUV-), Museo Nacional de Ciencias Naturales de Madrid (MNCN-), Museo Geominero de Madrid (MGM-). El material tipo de las tres especies que se discuten luego se conserva en la colección del Departamento de Geología (área de Paleontología), de la Universidad de Oviedo. La localización de los yacimientos se puede encontrar en Pardo y García-Alcalde (1984), Pardo Alonso (1997), Pardo Alonso y Gozalo Gutiérrez (1999), y Gozalo et al. (2002).

Para la morfología de la concha, especialmente la ornamentación, se utilizaron los términos morfológicos recomendados por Williams y Brunton (1997), en la edición revisada del T.I.P.

PHYLUM BRACHIOPODA Duméril, 1806 SUBPHYLUM RHYNCHONELLIFORMEA Williams et al., 1996

CLASE STROPHOMENATA Williams et al., 1996

ORDEN STROPHOMENIDA Öpik, 1934

Superfamilia Strophomenoidea King, 1846

Familia Douvillinidae Caster, 1939

Subfamilia Douvillininae Caster, 1939

Género Douvillina Oehlert, 1887

Especie-tipo: Orthis dutertrii Murchison, 1840.

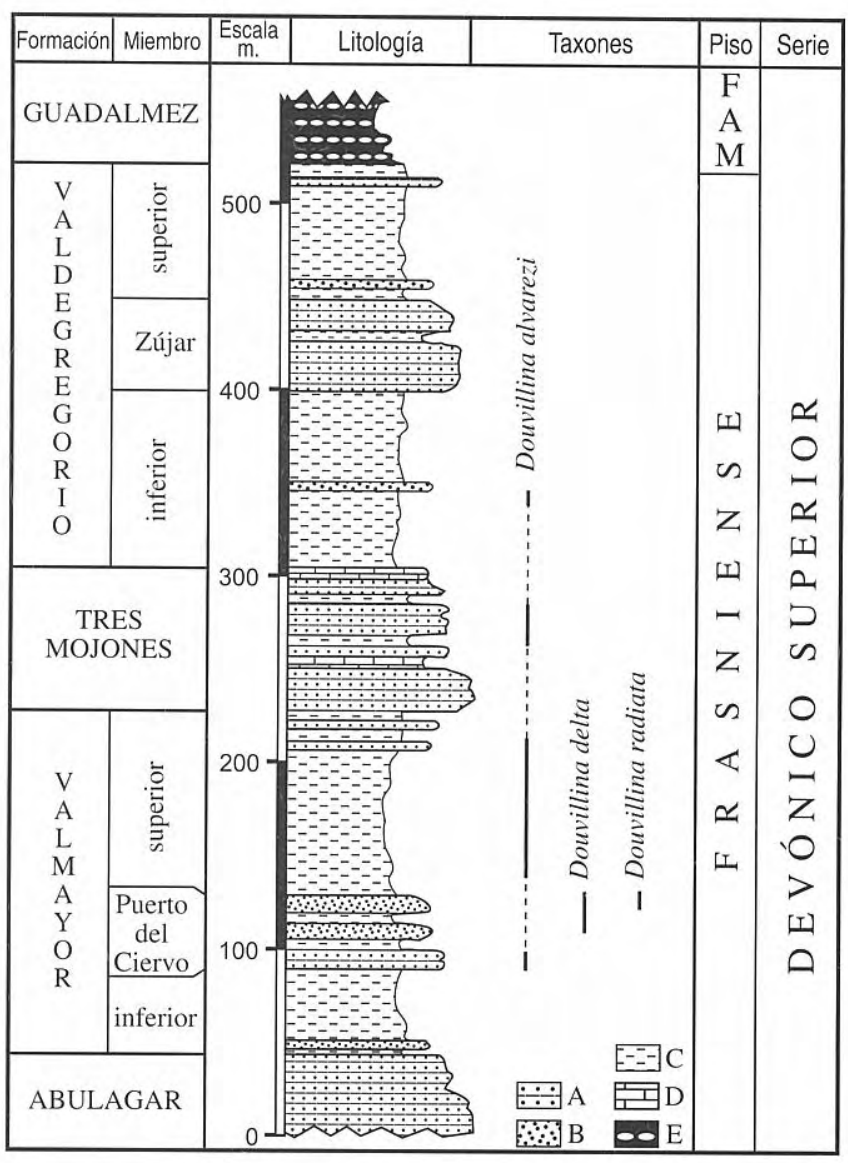

Figura 2. Columna estratigráfica sintética del Frasniense de la parte media de la ZCI meridional, y distribución estratigráfica de las especies del género Douvillina. Se han excluido las unidades vulcano-detríticas del sinclinal de Almadén. Fam: Fameniense. A: ortocuarcitas. B: areniscas. C: limolitas y pelitas. D: calizas bioclásticas. E: pelitas con nódulos.

Stratigraphic distribution of the Douvillina species in the middle part of the southern CIZ Frasnian. Almaden Syncline, vulcano-sedimentary units have been omitted. Fam: Famennian. A: orthoquartzites. B: sandstones. C: siltstones and shales. D: bioclastic limestones. E: shales with concretions.

\section{Diagnosis (enmendada aquí)}

Perfil lateral más o menos cóncavo-convexo, incluso plano-convexo; ornamentación parvicostulada; dentículos desarrollados sobre la mayor parte de la charnela; campo muscular ventral, subcircular, limitado por elevaciones curvadas y altas. Lóbulos del proceso cardinal erectos; septos laterales dorsales divergentes, desarrollando anteriormente una cavidad entre ellos.

Strongly to gently concave-convex, even plano-convex lateral profile; ornament parvicostellate; denticles developed over most of the hinge; high, very curved muscle field bounding ridges. Subcircular, relatively small ventral muscle field; erect cardinal process lobes; diverging dorsal side septa with a cavity developed between them anteriorly. 


\section{Discusión}

La nueva diagnosis que se propone es esencialmente la de Cocks y Rong (2000: p. 266), pero ampliando el tipo de perfil lateral para incluir formas fuerte a débilmente cóncavo-convexas, incluso plano-convexas.

Según la diagnosis enmendada de Harper y Boucot (1978), el género Douvillina quedaría restringido a formas con perfil fuertemente cóncavo-convexo y ornamentación desigualmente parvicostulada. Sin embargo, en las especies clásicamente asignadas al género, la ornamentación es mucho más variable; de hecho, gran parte de ellas se ajustan más a patrones regularmente parvicostulados que a desigualmente parvicostulados, como ocurre en la especie-tipo del género, D. dutertrei, y en las especies de Almadén. Por ello, en este caso, preferimos seguir el criterio de Rong y Cocks (1994) y Cocks y Rong (2000) que rechazaron pequeñas diferencias en la ornamentación como criterio útil de distinción genérica en la superfamilia Strophomenoidea, considerando más fiables grandes cambios de morfología de las valvas, como la resupinación o la geniculación. De todos modos, este último criterio debe tomarse con precaución, pues en Douvillina la geniculación está presente, pero sólo en algunas especies.

Otra cuestión es el perfil o convexidad de las valvas. Tanto la diagnosis de Harper y Boucot (op. cit.) como la de Cocks y Rong (op. cit.) de Douvillina, inciden en la presencia de perfil fuertemente cóncavo-convexo, lo cual dejaría fuera del género varias especies totalmente similares en el resto de caracteres, pero con perfil más aplanado. Sin embargo, muchos autores no conceden excesiva importancia a este carácter, admitiendo márgenes más amplios de variación. Por ejemplo, Brice (1988) mantiene en el género Douvillina a las especies Leptaena cedulae Rigaux, 1872, Douvillina douxami Rigaux, 1908, L. ferquensis Rigaux, 1872 y D. thomasi Rigaux, 1908, todas ellas débilmente cóncavo-convexas; $\mathrm{y}$, en el mismo trabajo, la autora redescribe la especietipo del género, Douvillina dutertrei, señalando la amplia variabilidad de curvatura de la valva ventral, desde formas casi hemisféricas (relación longitud desarrollada/longitud de la valva: $\mathrm{Lde} / \mathrm{L}=1,47$ ) hasta otras suavemente convexas $(\mathrm{Lde} / \mathrm{L}=1,07)$. Finalmente, Pardo y García-Alcalde (1984) incluyeron también en Douvillina la nueva especie $D$. radiata, de perfil suavemente cóncavo-convexo.

Este punto de vista parece el más acertado porque impide una innecesaria multiplicación de nuevos taxones genéricos y específicos, y debería formalizarse, para lo que se propone justamente la enmienda arriba expresada, en el sentido de incluir en Douvillina formas con un margen muy amplio de variación en el perfil lateral.

\section{Douvillina alvarezi}

(Pardo y García-Alcalde, 1984)

Fig. 3 A-M

- 1964 Douvillina ferquensis (Rigaux 1873); Puschmann, 109-113; lám. 7, figs. 13-16; lám. 8, figs. 1-8.
- 1970 Douvillina cedulae (Rigaux); Puschmann, 321-323; tab. 2 ; fig. 8 .

V* 1984 Douvillinoides (?) alvarezi n. sp., Pardo y GarcíaAlcalde, 96-100; fig. 8 a-j. (con sinónimos anteriores).

v 1987 Douvillinoides (?) alvarezi; Pardo y García-Alcalde (en García Sansegundo et al.), 27.

v 1992 Douvillinoides (?) alvarezi Pardo y García-Alcalde; J.L. García-Alcalde (en Soldevila Bartolí), 374.

v 1994 Douvillinoides ? alvarezi; Pardo Alonso y GarcíaAlcalde, fig. 2.

v 1996 Douvillinoides ? alvarezi; Pardo Alonso y GarcíaAlcalde, 77; fig. 3.

v 1999 Douvillina alvarezi (Pardo y García-Alcalde, 1984); Pardo Alonso, 66; lám. 3, fig. 1.

v• 2002 Douvillina alvarezi (Pardo y García-Alcalde, 1984); Gozalo et al., 87, 89, 91.

Material y yacimientos:

Además del material consignado en la descripción original de la especie, se han recolectado más ejemplares y otros han sido identificados en colecciones de museos. Los nuevos ejemplares asignados a la especie y siglados son:

Del yacimiento QUIN-18: MGUV-1501-1, MGUV1502, MGUV-1503, MGUV-1504, MGUV-1505-1, MGUV1505-2, MGUV-1506-1, MGUV-1507 y MGUV-1508. Del yacimiento MP-30: MGUV-1437-3, MGUV-1438-2, MGUV-1439-2. Del yacimiento "Chillón": MGM-234D-1. Del yacimiento "Convento de Chillón": MNCN-I-9538-2. Todos del sinclinal de Almadén.

Del sinclinal de Herrera: MGUV-603-1, MGUV-603-2, MGUV-603-6, MGUV-603-8, MGUV-603-11, MGUV-60317, MGUV-603-19, MGUV-603-22, MGUV-604-1, MGUV-2095-5, MGUV-2097-2 y MGUV-2097-3 del yacimiento CS-1; MGUV-1169-1, MGUV-1169-2 del yacimiento $\mathrm{PH}-2$.

Del sinclinal de Guadalmez: MGUV-1208-1, MGUV1210-1, MGUV-1212-4 del yacimiento VS-6s; MGUV1434-2 del yacimiento NG-1; MGUV-1447 del yacimiento TRM-19; MGUV-1436-1, MGUV-1436-2 del yacimiento TRM-20.

De todo este conjunto, sólo dos ejemplares conservan la concha (MGUV-1434 y MGUV-1447) y los demás son moldes internos o externos.

Además, la especie se ha encontrado por primera vez en los yacimientos (material no siglado): EP-21, EP-22, EP-23, EP-24, PH-3 y VMY-25, del sinclinal de Herrera; MP-23, MP-41, MP-46 y MP-49 del sinclinal de Almadén; GO-2, TRM-21, TRM-22, TRM-23, TRM-29 y TRM-31, del sinclinal de Guadalmez; CBU-7s, del flanco norte de Pedroches, en Cabeza del Buey.

\section{Discusión}

En un trabajo anterior (Pardo Alonso, 1999), ya se asignó esta especie al género Douvillina, aunque sin más detalles. En el presente trabajo, se justifica dicha atribución.

En cambio, en la descripción original de la especie (Pardo y García-Alcalde, 1984), el perfil plano-convexo y ornamentación regularmente parvicostulada ("multicostu- 
Figura 3. A - M. Douvillina alvarezi (Pardo y García-Alcalde, 1984). A, réplica en látex del holotipo DPO-16731, interior de valva ventral; yacimiento F-203, sinclinal de Almadén; base del Mb. Puerto del Ciervo, de la Fm. Valmayor. B, réplica en látex del paratipo DPO-16733, interior de valva dorsal, de la misma procedencia. C, réplica en látex del paratipo DPO-16743-III, exterior de valva ventral; yacimiento V-212, sinclinal de Almadén; base del Mb. Puerto del Ciervo, de la Fm. Valmayor. D, molde interno ventral, ejemplar MGUV-1505-1; yacimiento Quin-18, sinclinal de Almadén; miembro superior de la Fm. Valmayor. E - F, réplica en látex del ejemplar MGM-234D-2 y detalle de la misma; interior de valva ventral; localidad "Chillón", sinclinal de Almadén; probablemente del Mb. superior de la Fm. Valmayor. G, réplica en látex del ejemplar MGUV-2091, interior de valva ventral; yacimiento CS-1, sinclinal de Herrera del Duque; Mb. superior de la Fm. Valmayor. H, réplica en látex del ejemplar MGUV-603-2; exterior de valva ventral; misma procedencia que el anterior. I - M, moldes interno (I) y externo (L) de una valva dorsal, y réplicas en látex de ambos (J, K y M); ejemplar MGUV 1208-1; yacimiento VS-6s, sinclinal de Guadalmez; Fm. Tres Mojones. N - S. Douvillina delta Pardo y García-Alcalde, 1984. N, réplica en látex del holotipo DPO-16749-I, interior de valva ventral; yacimiento S-17a, sinclinal de Almadén. Mb. Puerto del Ciervo, de la Fm. Valmayor. O, réplica en látex del paratipo DPO-16752-I, interior de valva dorsal; misma procedencia que el anterior. P - Q, molde externo dorsal en vista lateral (P) y réplica en látex del molde interno, del paratipo DPO-16756; misma procedencia que el anterior. R, molde interno ventral, paratipo DPO-16749-IV; misma procedencia que el anterior. S, réplica en látex del paratipo DPO-16749-II, exterior de valva ventral; yacimiento S-17a, sinclinal de Almadén. Mb. Puerto del Ciervo, de la Fm. Valmayor. T - X. Douvillina radiata Pardo y García-Alcalde, 1984. T, réplica en látex del holotipo DPO-16759-I, interior de valva ventral; yacimiento S-17b, sinclinal de Almadén. Mb. Puerto del Ciervo, de la Fm. Valmayor. U, réplica en látex del paratipo DPO-16760-III, interior de valva dorsal; misma procedencia que el anterior. V, réplica en látex del paratipo DPO-16759-I, exterior de valva dorsal; misma procedencia que el anterior. W, réplica en látex de los paratipos DPO-16760-I (izquierda) y DPO-16760-IV, interiores dorsal y ventral respectivamente; misma procedencia que el anterior. $\mathbf{X}$, molde interno ventral del paratipo DPO-16761; misma procedencia que el anterior. La longitud de la escala gráfica es de $5 \mathrm{~mm}$.

A - M. Douvillina alvarezi (Pardo and García-Alcalde, 1984). A, latex cast from the holotype DPO-16731, ventral valve interior; sample F-203, Almadén syncline; base of the Puerto del Ciervo Member (Valmayor Formation). B, latex cast from the paratype DPO-16733, dorsal valve interior; from the same sample. C, latex cast from the paratype DPO-16743-III, ventral valve exterior; sample V-212, Almadén syncline; base of the Puerto del Ciervo Member (Valmayor Formation). D, inner ventral cast (specimen MGUV-1505-1); sample Quin-18, Almadén syncline; upper member of the Valmayor Formation. $\boldsymbol{E}-\boldsymbol{F}$, inner ventral latex cast (specimen MGM-234D-2), and close view; location "Chillón”, Almadén syncline; probably from the upper member of the Valmayor Formation. $\boldsymbol{G}$, inner ventral latex cast (specimen MGUV-2091); sample CS-1, Herrera del Duque syncline; upper member of the Valmayor Formation. H, outer ventral latex cast (specimen MGUV-603-2); from the same location than G. I - M, inner dorsal cast (I), outer cast (L), and correspondant latex casts (J, K and L) (specimen MGUV 1208-1); sample VS-6s, Guadalmez syncline; Tres Mojones Formation. N - S. Douvillina delta Pardo and García-Alcalde, 1984. N, latex cast from the holotype DPO-16749-I, ventral valve interior; sample S-17a, Almadén syncline. Puerto del Ciervo Member (Valmayor Formation). O, latex cast from the paratype DPO-16752-I, dorsal valve interior; from the same sample than $N . P-Q$, outer dorsal cast in lateral view $(P)$ and inner latex cast from the paratype DPO16756; from the same sample than N. R, inner ventral cast from the paratype DPO-16749-IV; from the same location than N. S, latex cast from the paratype DPO-16749-II, ventral valve exterior; from the same sample. $\boldsymbol{T}-\boldsymbol{X}$. Douvillina radiata Pardo and García-Alcalde, 1984. T, latex cast from the holotype DPO-16759-I, ventral valve interior; sample S-17b, Almadén syncline; Puerto del Ciervo Member (Valmayor Formation). U, latex cast from the paratype DPO-16760-III, dorsal valve interior; from the same sample than T. V, latex cast from the paratype DPO16759-I, dorsal valve exterior; from the same sample than T. W, latex casts from the paratypes DPO-16760-I (left) and DPO-16760-IV, dorsal and ventral interiors respectively; from the same sample than T. X, inner ventral cast from the paratype DPO-16761; from the same sample than T. The scale bar is $5 \mathrm{~mm}$ long.

lada", en la primera descripción) llevó a los autores a incluirla en el género Douvillinoides, con un punto de interrogación debido a la ausencia de ciertos caracteres comunes a las especies de este género, como una suave resupinación, plataforma ventral poco diferenciada, interárea dorsal anaclina y lóbulos del proceso cardinal dirigidos posteroventralmente.

Sin embargo, la morfología del interior de la concha es claramente la propia del género Douvillina, por lo que resulta más sensato, en el contexto de la enmienda propuesta en este trabajo, considerar el perfil lateral como un carácter más variable de lo que se había sugerido, antes que proponer un nuevo género para la especie. Esta decisión resulta reforzada por el hecho de que la propia especie-tipo del género, $D$. dutertrei, comprende formas con un perfil muy variable, incluso suavemente cóncavoconvexas. Como se verá a continuación (en "Comentarios"), se encontraron ejemplares más modernos que los de la serie tipo de D. alvarezi, débilmente cóncavo-convexos, que se asignan a la misma especie.

La ornamentación parvicostulada es, por su parte, un carácter que muestran algunas de las especies de Douvillina, según la diagnosis propuesta por Cocks y 

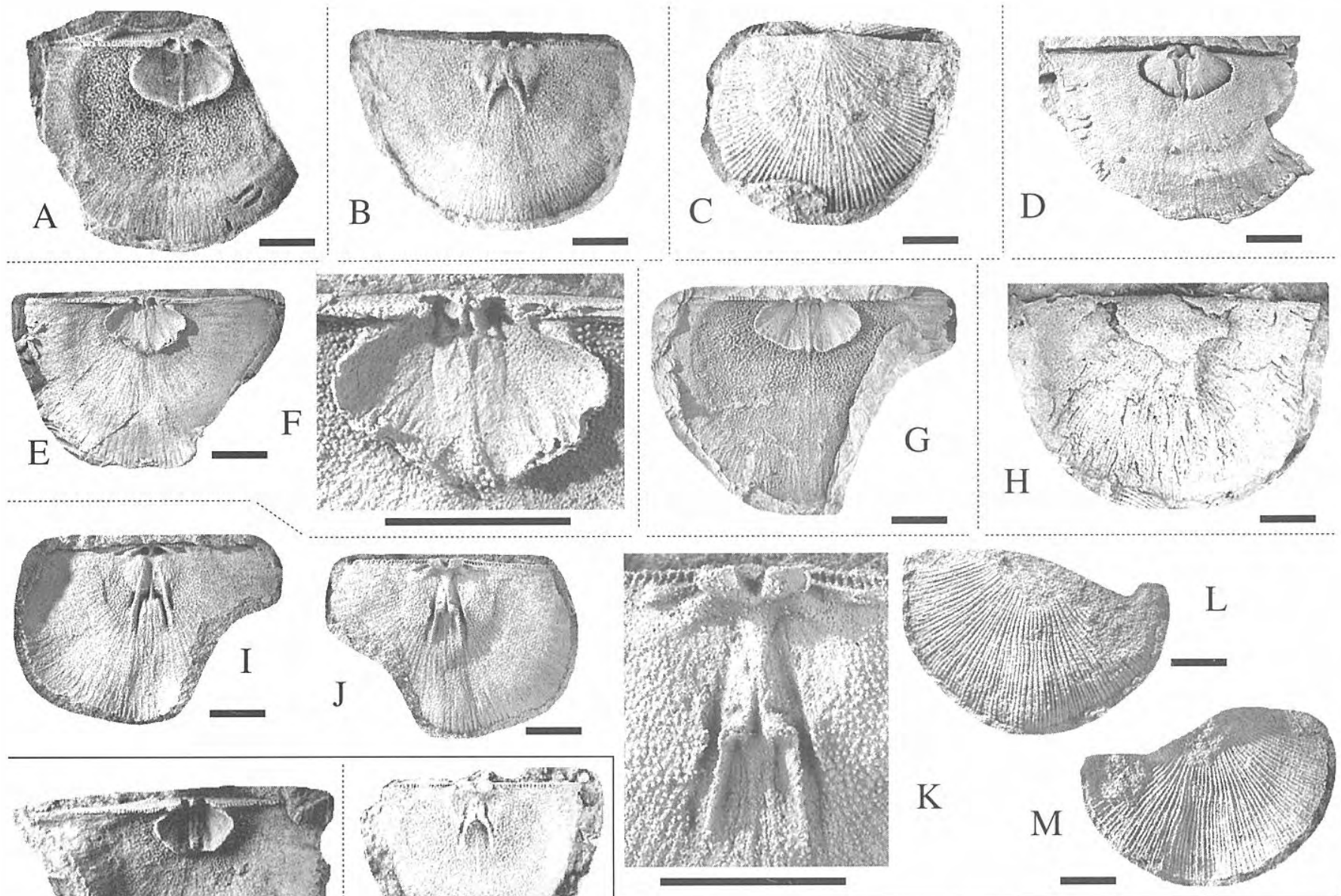

\section{L}

K
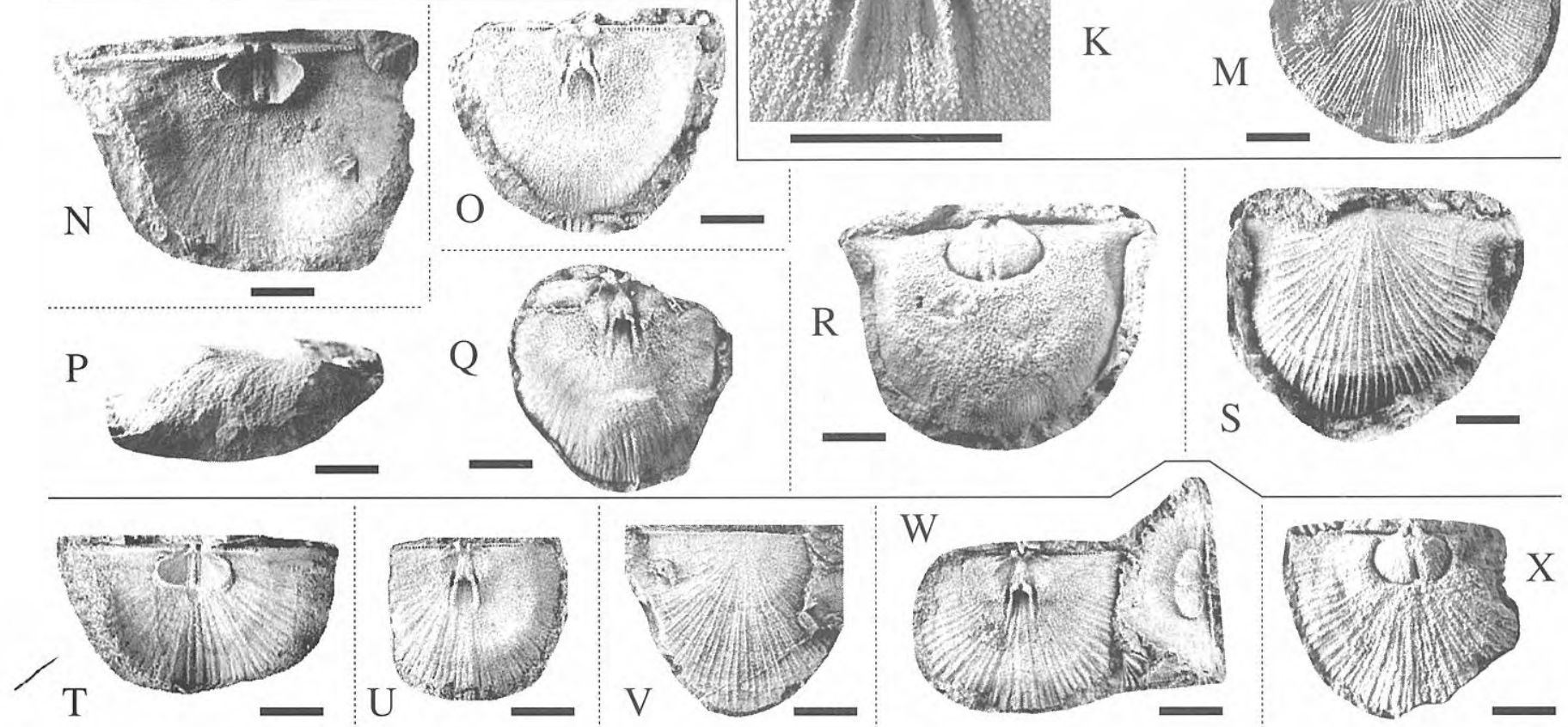

Rong (2000), de manera que, desde este punto de vista, igualmente $D$. alvarezi encaja, sin problemas, en el mismo género.

\section{Comentarios}

En principio, la especie parecía restringida a determinados niveles de la base de la Unidad 2 (actualmente Mb. Puerto del Ciervo); por encima, en la parte alta de la Fm. Valmayor, había restos de braquiópodos asignables a Douvillina, pero el estado fragmentario y la deformación del material no permitían una determinación más precisa. El nuevo material, mejor conservado, que se encontró en la Fm. Tres Mojones en Guadalmez, y en la Fm. Valmayor, en Herrera del Duque, así como en la parte oriental del sinclinal de Almadén (en su mayoría del yacimiento QUIN-18; ver Gozalo et al., 2002), corresponde también a $D$. alvarezi, aunque presenta pequeñas variaciones morfológicas respecto al material tipo de la especie. Así, los ejemplares de ambos conjuntos tienen ornamentación exterior regularmente parvicostulada, con elementos radiales de grosor comparable e interespacios similares; sin embargo, los ejemplares del material tipo son algo más pequeños, y su valva dorsal es plana, con una suave depresión media, 
mientras que los otros ejemplares la tienen suavemente cóncava, a veces con una débil geniculación cerca del extremo anterior. En estos ejemplares más modernos, la depresión media se acentúa en ocasiones, formando un seno amplio y poco profundo (Fig. $3 \mathrm{M}$ ), la auriculación (Fig. $3 \mathrm{D}$ y H) es más fuerte que en el material tipo y las estructuras internas dorsales están algo menos desarrolladas. Los interiores ventrales son prácticamente iguales en ambos conjuntos, si bien los gránulos que tapizan la cavidad visceral son más débiles en la mayoría de los ejemplares del grupo más moderno.

La posesión de valvas más grandes puede representar una adaptación a medios con fondos blandos, encaminada a conseguir mayor sustentación. De hecho, los ejemplares más modernos de Douvillina alvarezi aparecen, con frecuencia, en niveles pizarrosos de la Fm. Valmayor donde, junto al chonétido Pradochonetes muelleri, constituyen las especies autóctonas dominantes (Gozalo et al., 2002).

En su tesis doctoral, Puschmann (1964) describió y figuró con el nombre de Douvillina ferquensis varios ejemplares procedentes de Herrera del Duque, que en una publicación posterior (Puschmann, 1970) citó como D. cedulae. A través de la descripción y figuras proporcionadas por Puschmann (1964), estos ejemplares se atribuyen, con seguridad, a D. alvarezi.

\section{Distribución estratigráfica y geográfica}

Los nuevos hallazgos amplían la distribución de la especie respecto a la indicada en la descripción original (Pardo y García-Alcalde, 1984). Su primera aparición se produce en los niveles basales del Mb. Puerto del Ciervo, en areniscas cuarcíticas muy puras, típicas en la parte nororiental del sinclinal de Almadén; allí la especie se encuentra formando abundantes concentraciones de conchas, junto con otras especies, como Eoschuchertella jordani García-Alcalde (en Martínez-Chacón y García-Alcalde, 1978), caracterizando la Biozona de Extensión Coincidente D. alvarezi-E. jordani, de Pardo y García-Alcalde (1984); en una posición estratigráfica similar, pero en Herrera del Duque, se han encontrado ejemplares mal conservados que se consideran como Douvillina cf. alvarezi. La especie no se ha encontrado en la parte media-alta del Mb. Puerto del Ciervo, pero reaparece en los niveles lutíticos superiores de la Fm. Valmayor, en prácticamente toda la parte central de la ZCI meridional; también se encuentra con relativa frecuencia en la Fm. Tres Mojones, y alcanza en algunos puntos la Fm. Valdegregorio. Esta mayor extensión en la distribución estratigráfica supone también una ampliacion en términos de edad; así, con lo observado en el sinclinal de Almadén, ahora abarcaría desde la parte alta de la Zona de conodontos Mesotaxis asymmetricus Inferior (equivalente a la Zona de Palmatolepis transitans en la zonación actual), hasta al menos (Gozalo et al., 2002) la de M. asymmetricus Superior (equivalente grosso modo a la Zona de P. hassi Inferior). No se descarta que en los últimos niveles de aparición en otros sinclinales (Fm. Valdegregorio) la especie pueda alcanzar zonas todavía más modernas; de hecho, el Complejo Vulcano-Sedimentario de Chillón, del sinclinal de Almadén, que es en parte correlacionable con la Fm.
Valdegregorio, contiene conodontos que llegarían hasta la Biozona de Palmatolepis gigas Superior (es decir, la actual Palmatolepis linguiformis), de acuerdo con García-López et al. (1999).

Fuera de esta parte central de la ZCI meridional, Soldevila Bartolí (1992), con determinaciones de J.L. García-Alcalde, indicó su presencia en la Sierra de San Pedro (yac. ABQ-89 de este autor), concretamente en materiales procedentes de la Unidad de la Graña, correlacionable con la parte alta de la Fm. Valmayor y/o la Fm. Tres Mojones.

Con estos nuevos hallazgos, la extensión geográfica de la especie se amplía a todo el ámbito meridional central de la ZCI (desde el sinclinal de Herrera hasta el flanco norte del de Pedroches), llegando también por el oeste a la Sierra de San Pedro.

\section{Douvillina delta Pardo y García-Alcalde, 1984} Fig. $3 \mathrm{~N}-\mathrm{S}$

* 1984 Douvillina delta n. sp., Pardo y García-Alcalde, 100-102; fig. 8, k-q (con sinónimos anteriores).

v 1987 Douvillina delta; Pardo Alonso y García-Alcalde (en García Sansegundo et al.), 27.

v 1994 Douvillina delta; Pardo Alonso y García-Alcalde, fig. 2.

v 1996 Douvillina delta; Pardo Alonso y García-Alcalde, 77; fig. 3.

v 1999 Douvillina delta Pardo y García-Alcalde, 1984; Pardo Alonso, 66; lám. 3, fig. 3.

\section{Comentarios}

Esta especie fue citada únicamente en el sinclinal de Almadén, donde caracteriza, junto a $D$. radiata y Apousiella almadenensis Pardo y García-Alcalde, 1984, los niveles areniscosos de la parte media y superior del Mb. Puerto del Ciervo. En el flanco norte del sinclinal de Almadén, aparece también en los nuevos yacimientos NAL-2 y NAL-8. Es una especie muy característica, de perfil fuertemente cóncavo-convexo y geniculado (Fig. 3 P y Q), talla media a grande y ornamentación regularmente parvicostulada, o a veces desigualmente parvicostulada (Fig. $3 \mathrm{~S}$ ); estos caracteres la distinguen de las otras dos especies de Douvillina de la región.

\section{Douvillina radiata Pardo y García-Alcalde, 1984 Fig. 3 T-X}

* 1984 Douvillina radiata n. sp., Pardo y García-Alcalde, 102; fig. 8 r-t; fig. 9 a-c. (con sinónimos anteriores).

v 1987 D. radiata; Pardo y García-Alcalde (en García Sansegundo et al.), 27.

v 1994 Douvillina radiata; Pardo Alonso y GarcíaAlcalde, fig. 2.

v 1996 Douvillina radiata; Pardo Alonso y GarcíaAlcalde, 77; fig. 3. 
v 1999 Douvillina radiata, Pardo y García-Alcalde, 1984; Pardo Alonso, 66; lám. 3, fig. 2.

\section{Comentarios}

La talla pequeña, el aspecto característicamente radiado del interior de las valvas (Fig. $3 \mathrm{~T}, \mathrm{U}, \mathrm{W}$ y X) y el perfil suavemente cóncavo-convexo, la diferencian de las otras especies de Douvillina de la región.

En la descripción original de la especie ya se indicó (Pardo y García-Alcalde, 1984: p. 104) que a pesar del perfil suavemente cóncavo-convexo, el resto de los caracteres la situaban en Douvillina. La ornamentación desigualmente parvicostulada (Fig. 3 V), la ausencia de resupinación y la morfología de las estructuras cardinales la alejaban claramente de Douvillinoides, y tampoco presentaba los caracteres típicos de Douvillinaria, especialmente su perfil biconvexo.

Igual que en el caso de $D$. delta, los únicos hallazgos de esta especie se situaban en el sinclinal de Almadén, característicamente en los afloramientos de la parte alta del Mb. Puerto del Ciervo. Con posterioridad se encontró también en el sinclinal de Guadalmez (yacimiento GT33) en materiales de la misma unidad.

\section{AGRADECIMIENTOS}

El autor quiere agradecer a la Dra. Carmen Diéguez, del Museo Nacional de Ciencias Naturales de Madrid, y a la Dra. Isabel Rábano, directora del Museo Geominero (IGME, Madrid), el préstamo de los ejemplares de Douvillina de la ZCI, guardados en las colecciones de sus respectivas instituciones. También son de agradecer la detallada revisión y los comentarios realizados por los Dres. Jenaro L. GarcíaAlcalde, Fernando Álvarez y María Luisa Martínez Chacón, de la Universidad de Oviedo, que ayudaron a mejorar el manuscrito. Este trabajo es una contribución al Proyecto PB98-1542 financiado por la Dirección General de Estudios Superiores, y también al proyecto I.G.C.P. n ${ }^{\circ} 421$ North Gondwana Mid-Palaeozoic Bioevent/Biogeography patterns in relation to crustal dynamics.

\section{BIBLIOGRAFÍA}

Brice, D. 1988. Brachiopodes du Dévonien de Ferques (Boulonnais-France). Biostratigraphie du Paléozoïque, 7, 323-395.

Caster, K. E. 1939. A Devonian fauna from Colombia. Bulletin of American Paleontology, 24, 1-218.

Cocks, L.R.M. and Rong, J. Y. 2000. Strophomenida. Treatise on Invertebrate Paleontology. Part $\mathrm{H}$, Brachiopoda (Revised) Vol. 2. The Geological Society of America and The University of Kansas. Boulder and Lawrence, 219-348.

García-López, S., Sanz López, J. y Pardo Alonso, M. V. 1999. Conodontos (bioestratigrafía, biofacies y paleotemperaturas) de los sinclinales de Almadén y Guadalmez (Devónico-Carbonífero Inferior), Zona Centro-ibérica meridional, España. Revista Española de
Paleontología, $\mathbf{n}^{\mathbf{0}}$ extr. Homenaje al Prof. J. Truyols, 161-172.

García Sansegundo, J., Lorenzo Álvarez, S. y Ortega Gironés, E. 1987. Mapa y memoria explicativa de la Hoja Almadén (808) del Mapa geológico de España a escala 1:50.000. Instituto Geológico y Minero de España, 60 pp.

Gozalo, R., Pardo Alonso, M.V. y Valenzuela Ríos, J.I. 2002. Estratigrafía y Paleontología del yacimiento QUIN-18 (Formación Valmayor, Frasniense, sinclinal de Almadén, España). Revista Española de Paleontología, 17, 83-99.

Harper, C. W. Jr., and Boucot, A. J. 1978. The Stropheodontacea. Part. II: Douvillinidae, Telaeoshaleriidae, Amphistrophiidae and Shaleriidae. Palaeontographica, A, 161, 119-175.

Martínez-Chacón, M.L. y García-Alcalde, J.L. 1978. La génesis del koskinoide en braquiópodos articulados. Revista de la Facultad de Ciencias, Oviedo, 17-19, 261278.

Murchison, R. I. 1840. Description de quelques unes des coquilles fossiles les plus abondantes dans les couches devoniennes du Bas-Boulonnais. Bulletin de la Societé géologique de France, 11 (2), 250-256.

Oehlert, D. P. 1887. Études sur quelques fossiles dévoniens de l'Ouest de la France. Bibliothèque de l'École des Hautes Études, Sections des Sciences Naturelles, 33 (1), $80 \mathrm{pp}$.

Pardo, M. V. y García-Alcalde, J. L. 1984. Biostratigrafía del Devónico de la región de Almadén (Ciudad Real, España). Trabajos de Geología, Universidad de Oviedo, 14, 79-120.

Pardo Alonso, M. V. 1997. El Devónico meridional de la Zona Centroibérica. Tesis Doctoral, Universidad de Oviedo, 472 pp. (inédito).

Pardo Alonso, M. V. 1999. Invertebrados marinos del Paleozoico Superior de Castilla - La Mancha. In: La huella del pasado: Fósiles de Castilla - La Mancha (Coords. E. Aguirre e I. Rábano). Patrimonio histórico. Arqueología - Castilla La Mancha, Toledo, 51-73.

Pardo Alonso, M. V. y García-Alcalde, J. L. 1994. El Devónico de la Zona Centroibérica. Comunicaciones $X$ Jornadas Paleontología, Madrid, 153-156.

Pardo Alonso, M. V. y García-Alcalde, J. L. 1996. El Devónico de la Zona Centroibérica. Revista Española de Paleontología, $\mathbf{n}^{\mathbf{0}}$ extraordinario, 72-81.

Pardo Alonso, M. V. y Gozalo Gutiérrez, R. 1999. Historia de los estudios paleontológicos en el Devónico de la región de Almadén (Zona Centroibérica, España): periodo 1834-1990. Revista Española de Paleontología, $\mathbf{n}^{\mathbf{0}}$ extr. Homenaje al Prof. J. Truyols, 217-228.

Puschmann, H. 1964. Stratigraphie der Devonmulde von Herrera del Duque (Extremadura/Spanien) mit Beschreibung der Brachiopoden. Dissertation Univërsitat Ruprechst-Karl, Heidelberg, 141 pp. (inédito).

Puschmann, H. 1970. Das Paläozoikum der Nördlichen Sierra Morena am Beispiel der Mulde von Herrera del Duque (Spanien). Geologie, 19, 309-329.

Rigaux, E. 1872. Notes pour servir à la géologie du 
Boulonnais. Description de quelques Brachiopodes du terrain Dévonien de Ferques. Mémoires de la Société Académique de Boulogne, Boulogne-sur-Mer, 5, 1-16.

Rigaux, E. 1908 Le Dévonien de Ferques et ses Brachiopodes. Boulogne-sur-Mer, 33 pp.

Rong, J. Y. and Cocks, L. R. M. 1994. True Strophomena and a revision of the classification and evolution of strophomenoid and "strophodontoid" brachiopods. Palaeontology, 37, 651-694.

Soldevila Bartolí, J. 1992. La sucesión paleozoica en el sinforme de la Sierra de San Pedro (Provincias de Cáceres y Badajoz, SO de España). Estudios Geológicos, 48, 363-379.

Williams, A. and Brunton, C. H. C. 1997. Morphological and anatomical terms applied to brachiopods. Treatise on Invertebrate Paleontology. Part $\mathrm{H}$, Brachiopoda (Revised) Vol. 1. The Geological Society of America and The University of Kansas. Boulder and Lawrence, 423-440.

Manuscrito recibido: 9 de noviembre, 2001 Manuscrito aceptado: 30 de enero, 2002 